\title{
Ideal $\boldsymbol{n}$-body correlations with massive particles
}

\author{
R. G. Dall ${ }^{1}$ A. G. Manning1 ' S. S. Hodgman' ${ }^{1}$, Wu RuGway ${ }^{1}$, K. V. Kheruntsyan ${ }^{2}$ and A. G. Truscott ${ }^{1 \star}$
}

In 1963 Glauber introduced the modern theory of quantum coherence ${ }^{1}$, which extended the concept of first-order (onebody) correlations, describing phase coherence of classical waves, to include higher-order ( $n$-body) quantum correlations characterizing the interference of multiple particles. Whereas the quantum coherence of photons is a mature cornerstone of quantum optics, the quantum coherence properties of massive particles remain largely unexplored. To investigate these properties, here we use a uniquely correlated ${ }^{2}$ source of atoms that allows us to observe $n$-body correlations up to the sixthorder at the ideal theoretical limit (n!). Our measurements constitute a direct demonstration of the validity of one of the most widely used theorems in quantum many-body theory-Wick's theorem ${ }^{3}$ - for a thermal ensemble of massive particles. Measurements involving $n$-body correlations may play an important role in the understanding of thermalization of isolated quantum systems ${ }^{4}$ and the thermodynamics of exotic many-body systems, such as Efimov trimers ${ }^{5}$.

Glauber's modern theory of optical coherence and the famous Hanbury Brown-Twiss effect ${ }^{6}$ were pivotal in the establishment of the field of quantum optics. Importantly, the definition of a coherent state required coherence to all orders, which for example distinguishes a monochromatic but incoherent thermal source of light from a truly coherent source such as a laser. Higher-order correlation functions therefore provide a more rigorous test of coherence.

Higher-order correlations, characterized by an $n$-body correlation function $g^{(n)}$, are of general interest and have been investigated in many fields of physics including astronomy ${ }^{6}$, particle physics $^{7}$, quantum optics ${ }^{8}$, and quantum atom optics 9 . In particular they have been a fruitful area of research in the field of quantum optics, where they have been used to investigate the properties of laser light, including heralded single photons ${ }^{10}$, and the statistics of parametric down-conversion sources ${ }^{11}$. State-of-the-art quantum optics experiments have measured photon correlation functions up to sixth order for quasi-thermal sources ${ }^{8}$, allowing the possibility of performing full quantum state tomography ${ }^{12}$.

Higher-order correlations experiments with massive particles are currently approaching the same level of maturity as with photons. So far, experiments have directly observed correlations up to fourth order with single-atom-sensitive detection techniques for ultracold atomic bosons ${ }^{9,13,14}$, and second-order correlations for an atomic source of fermions ${ }^{15}$ demonstrating the uniquely quantum mechanical property of atom-atom antibunching. Alternative, indirect techniques have also been employed to investigate higher-order correlations, including the measurements of twobody (photoassociation ${ }^{16}$ ) and three-body ${ }^{17}$ loss rates that are sensitive, respectively, to second- and third-order correlation functions. Interestingly, fermionic atom pairs $^{18}$ and fermionic antibunching ${ }^{19}$ have also been observed in the atomic shot noise of absorption images.

In 1963, Glauber predicted that the maximal value of the same-point normalized $n$-body correlation function $g^{(n)}$ for
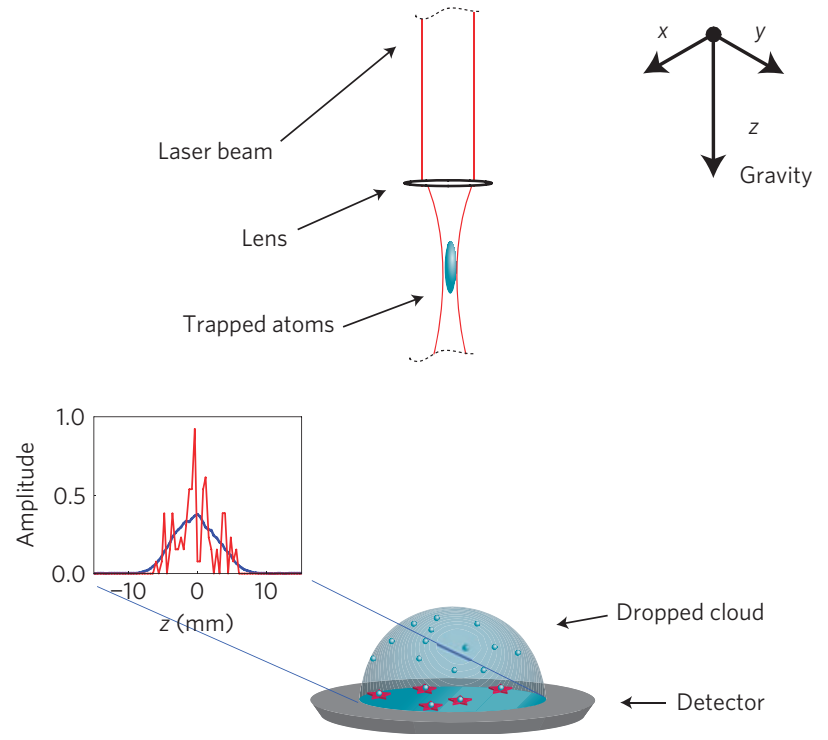

Figure 1 | Schematic of the experiment. An ultracold cloud of atoms is confined in a tightly focused laser beam. When released, the cloud drops $\sim 85 \mathrm{~cm}$, where its correlation properties are measured using a single-atom detector. Shown in the inset are the mean (dashed blue line) and a typical single (solid red line) longitudinal profile obtained in the experiment.

thermal light is directly related to the order of the function by a simple relationship $n$ ! (ref. 1). This $n$ ! dependence is a consequence of Wick's theorem ${ }^{3}$, which enables higher-order correlations to be expressed using products of one-body correlation functions. The applicability of Wick's theorem is not limited to just correlation functions for light, it has been also applied in many other fields; for example, it is commonly used in radioastronomy, nuclear physics ${ }^{7}$, and generally in quantum field theory ${ }^{3}$. The validity of Wick's theorem has been demonstrated with thermal photons, however, so far there have been no direct measurements demonstrating its validity to higher orders for massive particles.

The (unnormalized) two-body spatial correlation function $G^{(2)}\left(\mathbf{r}_{1}, \mathbf{r}_{2}\right)=\left\langle\hat{\Psi}^{\dagger}\left(\mathbf{r}_{1}\right) \hat{\Psi}^{\dagger}\left(\mathbf{r}_{2}\right) \hat{\Psi}\left(\mathbf{r}_{2}\right) \hat{\Psi}\left(\mathbf{r}_{1}\right)\right\rangle$ can be expressed as the probability of detecting two particles simultaneously at two particular locations $\mathbf{r}_{1}$ and $\mathbf{r}_{2}$, where $\hat{\Psi}^{\dagger}(\mathbf{r})$ and $\hat{\Psi}(\mathbf{r})$ are the field creation and annihilation operators. When normalized by the product of atomic densities $\rho\left(\mathbf{r}_{i}\right)=\left\langle\hat{\Psi}^{\dagger}\left(\mathbf{r}_{i}\right) \hat{\Psi}\left(\mathbf{r}_{i}\right)\right\rangle$ at respective locations, the two-body correlation function for a thermal source can be expressed mathematically in terms of the one-body correlation function $G^{(1)}\left(\mathbf{r}_{1}, \mathbf{r}_{2}\right)=\left\langle\hat{\Psi}^{\dagger}\left(\mathbf{r}_{1}\right) \hat{\Psi}\left(\mathbf{r}_{2}\right)\right\rangle$ :

$$
g^{(2)}\left(\mathbf{r}_{1}, \mathbf{r}_{2}\right)=1+\frac{\left|G^{(1)}\left(\mathbf{r}_{1}, \mathbf{r}_{2}\right)\right|^{2}}{\rho\left(\mathbf{r}_{1}\right) \rho\left(\mathbf{r}_{2}\right)}
$$

${ }^{1}$ Research School of Physics and Engineering, Australian National University, Canberra, Australian Capital Territory 0200, Australia, ${ }^{2}$ The University of Queensland, School of Mathematics and Physics, Brisbane, Queensland 4072, Australia. *e-mail: andrew.truscott@rsphysse.anu.edu.au 


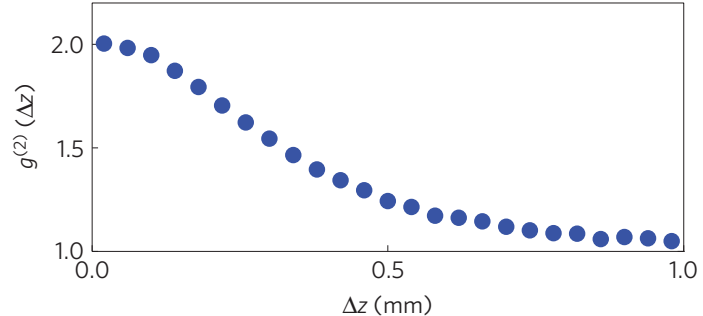

C

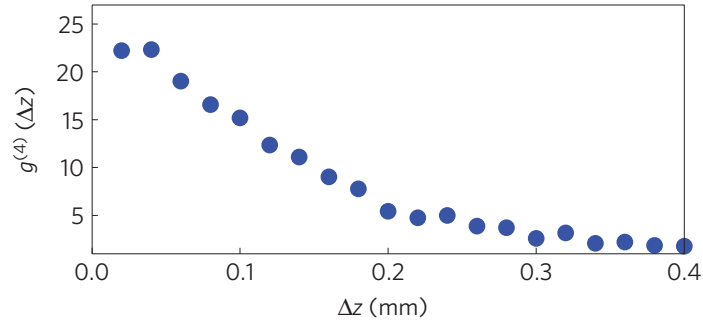

e

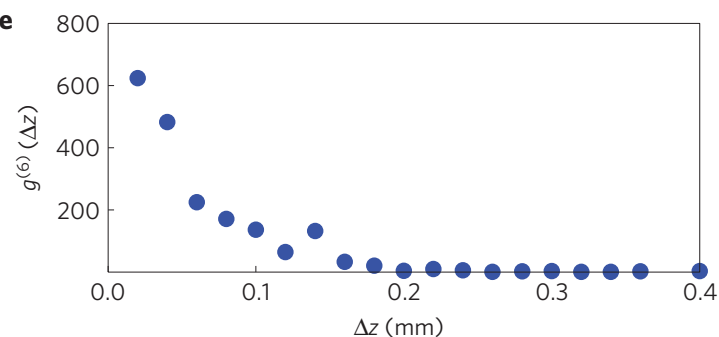

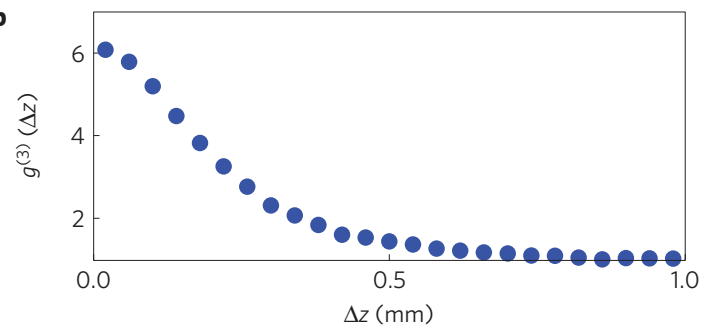

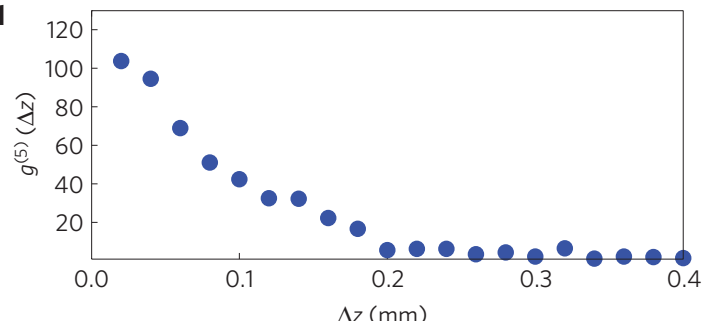

f

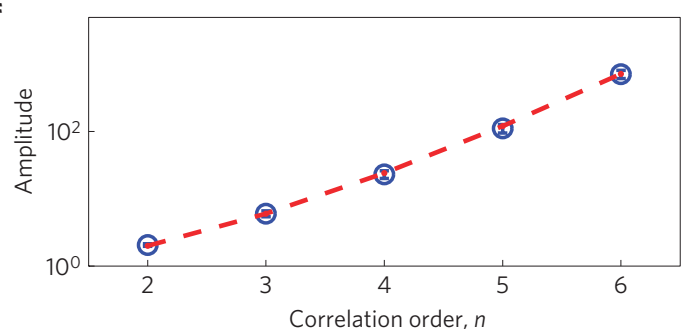

Figure 2 | Many-body correlation functions along the longitudinal direction (temporal dimension on the detector). The data is averaged over the $x$ and $y$ transverse directions using $\sim 1 \mathrm{~cm} \times 1 \mathrm{~cm}$ spatial bins. a-e, A Gaussian fit to the data yields the following peak correlation amplitudes: (a)

$g^{(2)}(0)=2.05 \pm 0.09$, (b) $g^{(3)}(0)=6.0 \pm 0.6$, (c) $g^{(4)}(0)=23 \pm 3$, (d) $g^{(5)}(0)=111 \pm 16$, and (e) $g^{(6)}(0)=710 \pm 90$. In f we show the $n$ ! scaling $($ dashed line) of the peak correlation amplitudes.

Likewise, the three-body correlation function can be written as a nested series, allowing it to be rewritten in terms of the lower-order correlation functions (Wick's theorem):

$$
\begin{aligned}
g^{(3)}\left(\mathbf{r}_{1}, \mathbf{r}_{2}, \mathbf{r}_{3}\right)= & 1+\frac{\left|G^{(1)}\left(\mathbf{r}_{1}, \mathbf{r}_{2}\right)\right|^{2}}{\rho\left(\mathbf{r}_{1}\right) \rho\left(\mathbf{r}_{2}\right)}+\frac{\left|G^{(1)}\left(\mathbf{r}_{2}, \mathbf{r}_{3}\right)\right|^{2}}{\rho\left(\mathbf{r}_{2}\right) \rho\left(\mathbf{r}_{3}\right)}+\frac{\left|G^{(1)}\left(\mathbf{r}_{3}, \mathbf{r}_{1}\right)\right|^{2}}{\rho\left(\mathbf{r}_{3}\right) \rho\left(\mathbf{r}_{1}\right)} \\
& +2 \operatorname{Re}\left(\frac{G^{(1)}\left(\mathbf{r}_{1}, \mathbf{r}_{2}\right) G^{(1)}\left(\mathbf{r}_{2}, \mathbf{r}_{3}\right) G^{(1)}\left(\mathbf{r}_{3}, \mathbf{r}_{1}\right)}{\rho\left(\mathbf{r}_{1}\right) \rho\left(\mathbf{r}_{2}\right) \rho\left(\mathbf{r}_{3}\right)}\right)
\end{aligned}
$$

Similarly Wick's theorem extends to higher orders, but we have omitted them for brevity.

Observing ideal bunching amplitudes requires the correlation length at the detector to be significantly larger than the detector resolution ${ }^{20}$, which for massive thermal particles has proved challenging, with the maximum bunching amplitudes reported in the literature being at most $\sim 20 \%\left[g^{(2)}(0) \approx 1.2\right]$ of the ideal value of $g^{(2)}(0)=1.2$ (ref. 21 ; see Supplementary Methods for the definition of the experimentally measured, volume integrated two-body correlation function at zero interparticle separation). To observe ideal bunching amplitudes, we have employed an ultracold cloud of partially transversely condensed ${ }^{2,22}{ }^{4} \mathrm{He}^{*}$ atoms in a strongly confining optical dipole trap (Fig. 1). This unique cloud can produce ideal bunching amplitudes even with the limited resolution available with current delay-line detectors ( $\sim 100 \mu \mathrm{m}$ spatially, $\sim 1$ ns temporally). This is a result of the gas being transversely coherent, but longitudinally incoherent, where interference between the many longitudinal modes occurs over the entire transverse extent of the cloud.

The experiment is performed using a novel technique (Supplementary Information), where we produce ultracold clouds of ${ }^{4} \mathrm{He}^{*}$ in a highly anisotropic optical dipole trap, aligned with its weak axis in the direction of gravity ( $z$-axis). A simplified schematic of the experiment is shown in Fig. 1. The ultracold atomic cloud is at a temperature of just $\sim 60 \mathrm{nK}$ and contains $\sim 330$ atoms. To generate the required statistics, over 2,000 independent realizations of the experiment were undertaken. We measure spatial correlations after time-of-flight expansion, by dropping the clouds onto a singleatom-sensitive delay-line detector located $\sim 85 \mathrm{~cm}$ below the trap. An average many-body correlation function can then be calculated.

The major result of this paper is illustrated in Fig. 2, where our near-ideal many-body correlation functions $g^{(n)}(\Delta z) \equiv$ $g^{(n)}(\Delta z, \ldots, \Delta z)$ (Supplementary Information) up to sixth order are shown as a function of interparticle separation along the longitudinal direction. The peak amplitudes for all the orders are in good agreement with the maximum value of $n$ ! expected for the $n$th order correlation function $g^{(n)}(0)$ for a thermal gas.

To provide further detail on the three-body correlation function $g^{(3)}(\Delta z)$ in Fig. 2b, we have also plotted in Fig. 3 a full 2D surface plot of the $g^{(3)}\left(\Delta z_{1}, \Delta z_{2}\right)$-function to which $g^{(3)}(\Delta z)=g^{(3)}(\Delta z, \Delta z)$ is the diagonal cut along $\Delta z_{1}=\Delta z_{2}$. To interpret the surface in Fig. 3, one should realize that the three-body interference term is maximum when all three particles are close together (that is, $\Delta z_{1}=\Delta z_{2} \sim 0$ ). On the other hand, when one of the particles is taken to large separation (that is, $\Delta z_{i} \gtrsim 2 \mathrm{~mm}$ in our case), one recovers the two-body correlation function from this plot, along the remaining dimension. The complete fourth-, fifth-, and sixth-order correlation functions, $g^{(4)}\left(\Delta z_{1}, \Delta z_{2}, \Delta z_{3}\right)$, $g^{(5)}\left(\Delta z_{1}, \Delta z_{2}, \Delta z_{3}, \Delta z_{4}\right), g^{(6)}\left(\Delta z_{1}, \Delta z_{2}, \Delta z_{3}, \Delta z_{4}, \Delta z_{5}\right)$ require four-, five- and six-dimensional plots respectively, so for clarity we have plotted these in Fig. 2c-e similarly to $g^{(3)}$, that is, for equal spatial separations for all particles. 


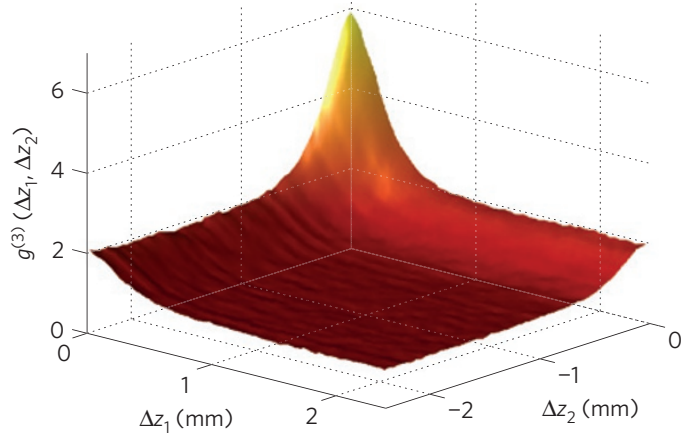

Figure 3 | Surface plot of the three-body correlation function $g^{(3)}\left(\Delta z_{1}, \Delta z_{2}\right)$.

For atomic systems this is the first reported measurement of the fifth- and sixth-order correlation functions and, importantly, the measurements of the lower-order correlations are up to two orders of magnitude greater in amplitude than previously reported ${ }^{13,14,21}$. Remarkably, the measurement of the six-body correlation function demonstrates that the probability of finding six particles at the same location is nearly three orders of magnitude greater than for large separations. Surprisingly, to our knowledge our near ideal sixth-order correlation measurement for massive thermal particles surpasses all measurements for thermal photons.

The peak bunching amplitudes (Fig. 2f) of the many-body correlation functions are in agreement with the expected $n$ ! dependence of Wick's theorem for thermal particles, thus confirming the validity of quantum theory of boson statistics for massive particles up to the sixth order ${ }^{1}$. Counter-intuitively, the signal to noise ratio is approximately unchanged for all orders, owing to the signal from the bunching amplitude rapidly increasing, which compensates for the increased statistical noise due to a reduction in the number of multi-particle interference events for the higher orders. Ultimately, our finite flux limits the order of the correlation function we can accurately produce. Note, that the two-body correlation function, Fig. 2a, directly yields the longitudinal correlation length of the gas. However, for correlation functions greater than two-body, Fig. 2b-e, a compression of the width is observed owing to our effective bin size increasing.

We emphasize that the nature of the critical transition to a transversely condensed gas, which enabled us to measure higherorder correlations at their ideal limit, is a quantum degeneracy driven (rather than interaction driven ${ }^{23}$ ) transition of an ideal Bose gas confined to a highly anisotropic trap. As discussed in refs 2,22, this occurs owing to the saturation of population in the transversely excited states-hence the term 'transverse condensation'. Our measurements of the transverse properties of the gas, including the fraction of the atoms in the transverse ground state and the transverse correlation functions (to be discussed elsewhere), are indeed in excellent agreement with the predictions of the theory for a harmonically trapped ideal Bose gas. On the other hand, the longitudinal properties of the gas, especially well below the critical temperature, are intermediate between the theory of a highly degenerate ideal Bose gas and a weakly interacting quasicondensate $^{24}$. Given the relatively small number of atoms in our clouds (a few hundred), we expect the finite-size effects to be significant, which means that the physics in the longitudinal dimension is dominated by broad crossovers between that of a pure non-interacting gas and of a weakly interacting gas approaching the quasicondensate regime. It is perhaps then not surprising that the measured longitudinal correlation length $(394 \mu \mathrm{m})$ is significantly larger than that predicted by ideal Bose gas theory $(95 \mu \mathrm{m})$, but shorter than that expected in the quasicondensate regime ( $\sim 850 \mu \mathrm{m}$; Supplementary Information). Nonetheless, we still expect the correlation functions we measure to satisfy the factorial relationship predicted by Wick's theorem, as the equalpoint momentum-momentum correlation function is given by $g^{(2)}(\mathbf{k}, \mathbf{k}) \approx 2$ for both the ideal Bose gas and the weakly interacting quasicondensate regime ${ }^{24}$.

In conclusion, we have measured near-ideal many-body correlation functions in a thermal ensemble of ultracold ${ }^{4} \mathrm{He}^{*}$ atoms and demonstrated the validity of Wick's theorem for massive particles to sixth order. Our results show that quantum atom optics experiments can now rival, and in some cases exceed, the performance of quantum optics experiments. The ability to accurately measure photon-photon correlations in quantum optics has been pivotal in enabling some of the foundational tests of quantum mechanics, such as violations of Bell's inequalities ${ }^{25,26}$ and the demonstration of Einstein-Podolsky-Rosen entanglement ${ }^{27}$. Our correlation measurements with ultracold ${ }^{4} \mathrm{He}^{*}$ atoms may pave the way for similar future quantum atom optics tests of the tenets of quantum mechanics for massive particles ${ }^{12}$. Moreover, higher-order correlations may play an important role as accurate probes of the $3 \mathrm{D}$ condensates ${ }^{28}$, as well as intriguing quantum states incorporating lower dimensions ${ }^{29}$ and other strongly correlated systems ${ }^{17}$. Finally, they may also provide unambiguous evidence of $p$ - and $d$-wave pairings ${ }^{30}$, which may offer valuable insights into high-temperature superconductivity.

Received 4 November 2012; accepted 15 April 2013; published online 19 May 2013

\section{References}

1. Glauber, R. The quantum theory of optical coherence. Phys. Rev 130, 2529-2539 (1963).

2. Van Druten, N. J. \& Ketterle, W. Two-step condensation of the ideal Bose gas in highly anisotropic traps. Phys. Rev. Lett. 79, 549-552 (1997).

3. Wick, G. C. The evaluation of the collision matrix. Phys. Rev. 80, 268-272 (1950).

4. Giraud, A. \& Serreau, J. Decoherence and thermalization of a pure quantum state in quantum field theory. Phys. Rev. Lett 104, 230405 (2010).

5. Kramer, T. et al. Evidence for Efimov quantum states in an ultracold gas of caesium atoms. Nature 440, 315-318 (2006).

6. Hanbury Brown, R. \& Twiss, R. Q. A test of a new type of stellar interferometer on Sirius. Nature 178, 1046-1048 (1956).

7. Baym, G. The physics of Hanbury Brown-Twiss intensity interferometry: From stars to nuclear collisions. Acta Phys. Polonica B 29, 1839-1883 (1997).

8. Avenhaus, M., Laiho, K., Chekhova, M. \& Silberhorn, C. Accessing higher order correlations in quantum optical states by time multiplexing. Phys. Rev. Lett. 104, 063602 (2010)

9. Yasuda, M. \& Shimizu, F. Observation of two-atom correlation of an ultracold neon atomic beam. Phys. Rev. Lett. 77, 3090-3093 (1996).

10. Uren, A. B., Silberhorn, C., Ball, J. L., Banaszek, K. \& Walmsley, I. A. Characterization of the nonclassical nature of conditionally prepared single photons. Phys. Rev. A 72, 021802 (2005).

11. Ivanova, O. A., Iskhakov, T. Sh., Penin, A. N. \& Chekhova, M. V. Multiphoton correlations in parametric down-conversion and their measurement in the pulsed regime. Quant. Electron. 36, 951-956 (2006).

12. Schilling, U., von Zanthier, J. \& Agarwal, G. Measuring arbitrary-order coherences: Tomography of single-mode multiphoton polarization-entangled states. Phys. Rev. A 81, 013826 (2010).

13. Hodgman, S. S, Dall, R. G., Manning, A. G., Baldwin, K. G. H. \& Truscott, A. G. Direct measurement of long-range higher-order coherence in Bose-Einstein condensates. Science 331, 1046-1049 (2011).

14. Guarrera, V. et al. Observation of local temporal correlations in trapped quantum gases. Phys. Rev. Lett. 107, 160403 (2011).

15. Jeltes, T. et al. Comparison of the Hanbury Brown-Twiss effect for bosons and fermions. Nature 445, 402-405 (2007).

16. Kinoshita, T., Wenger, T. \& Weiss, D. S. Local pair correlations in one-dimensional Bose gases. Phys. Rev. Lett 95, 190406 (2005).

17. Haller, E. et al. Three-body correlation functions and recombination rates for bosons in three dimensions and one dimension. Phys. Rev. Lett 107, 230404 (2011).

18. Greiner, M., Regal, C. A., Stewart, J. T. \& Jin, D. S. Probing pair-correlated fermionic atoms through correlations in atom shot noise. Phys. Rev. Lett. 94, 110401 (2005). 
19. Rom, T. et al. Free fermion antibunching in a degenerate atomic Fermi gas released from an optical lattice. Nature 444, 733-736 (2006).

20. Gomes, J. V. et al. Theory for a Hanbury Brown-Twiss experiment with a ballistically expanding cloud of cold atoms. Phys. Rev. A 74, 053607 (2006).

21. Dall, R. G. et al. Observation of atomic speckle and Hanbury Brown-Twiss correlations in guided matter waves. Nature Commun. 2, 291 (2011).

22. Armijo, J., Jacqmin, T., Kheruntsyan, K. V. \& Bouchoule, I. Mapping out the quasi-condensate transition through the dimensional crossover from one to three dimensions. Phys. Rev. A 83, 021605 (2011).

23. Bouchoule, I., Kheruntsyan, K. V. \& Shlyapnikov, G. V. Interaction-induced crossover versus finite-size condensation in a weakly interacting trapped 1D Bose gas. Phys. Rev. A 75, 031606 (2007).

24. Bouchoule, I., Arzamasovs, M., Kheruntsyan, K. V. \& Gangardt, D. M. Two-body momentum correlations in a weakly interacting one-dimensional Bose gas. Phys. Rev. A 86, 033626 (2012).

25. Aspect, A., Dalibard, J. \& Roger, G. Experimental test of Bell's inequalities using time-varying analyzers. Phys. Rev. Lett. 49, 1804-1807 (1982).

26. Rarity, J. G. \& Tapster, P. R. Experimental violation of Bell's inequality based on phase and momentum. Phys. Rev. Lett 64, 2495-2498 (1990).

27. Ou, Z., Pereira, F., Kimble, H. \& Peng, K Realization of the Einstein-Podolsky-Rosen paradox for continuous variables. Phys. Rev. Lett 68, 3663-3666 (1992).

28. Perrin, A. et al. Hanbury Brown and Twiss correlations across the Bose-Einstein condensation threshold. Nature Phys. 8, 195-198 (2012).
29. Manz, S. et al. Two-point density correlations of quasicondensates in free expansion. Phys. Rev. A 81, 031610 (2010).

30. Kitagawa, T., Aspect, A., Greiner, M. \& Demler, E. Phase-sensitive measurements of order parameters for ultracold atoms through two-particle interferometry. Phys. Rev. Lett. 106, 115302 (2011).

\section{Acknowledgements}

A.G.T and K.V.K. acknowledge the support of the Australian Research Council through the Future Fellowship grants FT100100468 and FT100100285.

\section{Author contributions}

S.S.H., R.G.D. and A.G.T. conceived the experiment. A.G.M., S.S.H. and W.R. collected the data presented in this Letter. K.V.K. developed the Bose model and provided theoretical insight into the results. All authors contributed to the conceptual formulation of the physics, the interpretation of the data and writing the manuscript.

\section{Additional information}

Supplementary information is available in the online version of the paper. Reprints and permissions information is available online at www.nature.com/reprints. Correspondence and requests for materials should be addressed to A.G.T.

\section{Competing financial interests}

The authors declare no competing financial interests. 